\title{
A review of life table construction
}

\begin{abstract}
Life table is the one of the oldest statistical techniques and is extensively used by medical statisticians and actuaries. It is also the scheme for expressing the form of mortality in terms of probabilities. The life table is constructed from census data and death registration data, it can also be constructed from vital registration or retrospective surveys. Generally, they are constructed for age, gender, ethnic groups and occupational groups and so on. Some classification of life table such as cohort or current life table which are classified according to the reference year, unabridged (complete) or abridge life table classified according to length of age interval and single or multiple decrement life table classified according to the number of characteristics considered were discussed in the article.
\end{abstract}

Volume 5 Issue 3 - 2017

\author{
Ilker Etikan, Sulaiman Abubakar, Rukayya \\ Alkassim \\ Near East University Faculty of Medicine Department of \\ Biostatistics, Nicosia-TRNC, Cyprus
}

\begin{abstract}
Correspondence: Ilker Etikan, Near East University Faculty of Medicine Department of Biostatistics, Nicosia-TRNC, Cyprus,
\end{abstract} Email ietikan@gmail.com

Received: November 24, 2016 | Published: March 02, 2017

Keywords: life tables, survival analysis, kaplan meirer

\section{Introduction}

A life table is a terse way of showing the probabilities of a member of a particular population living to or dying at a precise age. ${ }^{1}$ It is another effective way of expressing the death rates experienced by a population during a given period of time. ${ }^{2}$ The death rates were discussed and referred by many researchers as observe rates, but one of the problems of using these rates as levels of death rates is that the effect of age distribution of an actual population is reflected or requires the adoption of normal populations for acceptable comparison of levels of mortality in different populations. To overcome these problems, life table is used. Life table best expresses the pattern of human mortality. It presents a detailed sketch of a population that systematically gets depleted through death at each age.

In the year 1960, ketfitz defines life table as the scheme for expressing the form of mortality in terms of probabilities. He further states that it is also a model of a population covering the simplest case which is worth discussing: a cohort or group of people born at the same moment close to migration and followed through successive ages until they die. The life table has given its shape to the natural world like other successful models; researchers are incapable of thinking about population change and mortality for any other starting point". Sometimes, life table is referred to as the mortality table which is one form of combining the mortality rates of a population at different ages into a single statistical model. The statistical history or hypothetical cohort of persons born at an instant time, followed through till all members of the cohort are no more. The construction of generation life table tells about the general mortality trends existing among male and female populations or mortality status of people. ${ }^{3}$

\section{Historical background of life table}

Emlius macer was the person who first published the table of mortality or life table to the Roman Juris-consulate, the table dates about the year 225 A.D macer's table. A more correct schedule was developed by Ulpian a few decades later. The table was named ulpian's table and was considered more correct than that of macer because it reflect the actual expectancies. Girolamo cadano in 1957 proposed the life expectancy as a linear decreasing function of the age x. john garaunt in 1662 tabulated the number of deaths based in the study "bill of mortality" in 1658 on the city of London. This was basic life table which became the origin of the concept of life table as we know today. However, the life table was defective because it was based on mortality experience alone.
In 1693, the first mortality table is usually credited to Edmund halley who constructed a life table base on the data from Breslau city in Poland. The table thought to be the first and as well as best of its kind. Halley's table based on the data concerning birth and death during the year 1687 to 1691 which contained the most of the column of modern life table with new application extended to insurance, pension and annuities. The table became the accepted standard of its time and it could not be corrected because it was based on the assumption that the population had remained stationary which was not possible entirely.

In $17^{\text {th }}$ and $18^{\text {th }}$ century, several attempt where made for constructing a life table on the basis of limited or incomplete data. In 1746 Antoine de parcieux also contribute in the development and calculation of expectations. Milne in 1815 was the first to prepare and published a scientifically correct life table classified by age based on both population and death data.

\section{Classification of life table}

Life table can be classified as cohort or current life table according to the reference year of the table, unabridged (complete) or abridge life table according to length of age interval and single or multiple decrement life table according to the number of characteristics considered.

Cohort Life Table: A cohort life table is also called generation life table or longitudinal life table, because the cohort is constructed on the basis of sequence $\mathrm{Q}_{0}, \mathrm{Q}_{2}, \mathrm{Q}_{3}, \ldots, \mathrm{Q}_{\mathrm{n}}$. The cohort life table reflects the mortality experience of an real cohort from birth until no lives remain in the group. Its main advantage is its conceptual simplicity and in fact agrees with the definition of a life table but its major disadvantage is the very long term for which data are required and to which mortality risks are referred. The life span of a cohort represented by $\mathrm{n}$ can be anywhere near 100 years or more since the upper limit of human life is 100 years [4]. Therefore the study of the death sequence process till the last person is death in the cohort requires long period of waiting. This reason makes the cohort life table limited in use and suitable to study the mortality of plants and insect that have short life span [5].

Cohort working life tables were constructed for Canadian men and women aged 50 and older for corresponding period table comparison. The tables are obtained using single annual age time series of participation rates from the Canada Labour Force Survey master files of the Statistics for 1976-2006. The cohort calculations are based on stochastic projections of mortality joined with alternative assumptions 
of the future participation rates. Separate tables are prepared for the years 1976, 1991, and 2006, therefore spanning a period of significant advances in life expectancy and strong upward trends in female participation. ${ }^{6}$

Current Life Table: This is also referred to as cross sectional life table, period life table or specified life table. It employs data for a single cross section of time to represent an entire generation. In current life table, a sequence of $P_{0}, P_{1}, P_{2}, \ldots P_{w}$ depicting current mortality pattern of a given population is constructed to represent the death process $Q_{0}, Q_{2}, Q_{3}, \ldots, Q_{n}$ in a cohort. Its main advantage is it provides measures localized in time such as change in expectation of life at birth from one year to the next. Its main disadvantage is its conceptual complexity.

Complete Life Table: Complete life table contains data for every single years of age from birth to the last applicable age, it is also called unabridged life table. ${ }^{7}$ In order to economize space, sometime the basic values from a complete life table are presented only for every fifth age.

Abridge Life Table: Abridge table contains the data by intervals of five or ten-years, except in the initial years. This is the abridged life tables that most users frequently encounter.

Single Decrement Life Table: In this life table only one cause of death and only one characteristic are considered at a time, and are concerned with general experience of a cohort by age.

Multiple Decrement Life Table: This table described the separate and combined effects of more than one characteristic; it may consider more than one cause of death and or more than one characteristic at a time.

\section{Methodology}

The main purpose of life tables is to describe the mortality behavior of specific groups. Construction of general life tables is based on census and death statistics figures of local populations under the hypothesis of a closed demographic system. ${ }^{8}$ It's also a statistical table based on age-specific death rates in a specific population. Life tables have been used earlier as a measure of summarizing the health status of a group of individuals. It also identifies the death rates of a given time experienced by a population.

A typical life table generally has the following columns.

\begin{tabular}{lllllll}
\hline 1$]$ & {$[2]$} & {$[3]$} & {$[4]$} & {$[5]$} & {$[6]$} & {$[7]$} \\
\hline
\end{tabular}

$X$

Where the symbols entering in this able are defined below

The symbol $\mathbf{x}$ represent the exact age or it may refer to the age interval.

The survival at age $X$ refers to the $I_{X}$ implies to the persons living at any age $X$ in any year out an assumed number of birth $1_{0}$, which is the cohort or radix of the life table which is assumed number of births at age 0 and is usually taken as $100,000, I_{X}$ refers to the number of survivors.

Number of death $d_{X}$ is the number among the survival at age $\mathrm{x}$ persons who died before reaching the $(x+1)$, that is

$$
d_{x}=l_{x}-l_{x+1}
$$

Mortality rate $q_{X}$ is the probability the person at exact age will die with one year following of that age, this is obtain as follows.

$$
\begin{aligned}
q_{x}= & \frac{l_{x}-l_{x+1}}{l_{x}}=\frac{d_{x}}{l_{x}} \\
& =1-p_{x}
\end{aligned}
$$

Where $p_{x}$ is the probability of survivorship which implies the probability that person aged $X$ is surviving up to his next birthday $(x+1)$ which is

$$
p_{x}=\frac{l_{x+1}}{l_{x}}
$$

Person-years lived $L_{x}$ is the number of year lived in the aggregate by the cohort $l_{x}$ persons between exact age $\mathrm{x}$ and exact age $X+1$ of persons alive at age $X$. It is also called the life table population. $L_{x}$ Will always be the same in each year under a stationary condition. When the death at an age $\mathrm{x}$ are assumed to be uniformly distributed, $L_{x}$ becomes the mid-year population which is given by

$$
\begin{aligned}
& L_{x}=l_{x}+0.5\left(l_{x}-l_{x+1}\right) \\
& L_{x}=0.5\left(l_{x}+l_{x+1}\right) \text { for } X \geq 2
\end{aligned}
$$

This assumed that a person dying between the age $X$ and $X+1$ , on an average, lives 0.5 years. Therefore we may also define $L_{x}$ as:

$$
L_{x}=l_{x}-0.5 d_{x}
$$

But the linearity assumption is not valid for age 0 and 1 , and in such cases the other approximate value of $L_{x}$ is:

$$
L_{x}=1 . l_{x}+0.3\left(l_{x}-l_{x}\right)=0.3 l_{x}+0.7 l_{x}
$$

This assumed that a person dying before the age of one year on an average lives 0.3 years.

Persons-years lived after $X\left(T_{X}\right)$ : This implies the number of years lived by the cohort $1_{x}$ after attaining the age $\mathrm{x}$. It is usually considered as the total future life table of the lx persons who reached age $X$. this is given as:

$$
\mathrm{T}_{\mathrm{x}}=\sum_{\mathrm{y}=\mathrm{x}}^{\mathrm{w}} \mathrm{L}_{\mathrm{y}}
$$

Where $\mathrm{w}$ is the highest age attainable which is

$$
T_{x}=L_{x}+L_{x+1}+L_{x+2}+\ldots+L_{w}
$$

Complete Expectation of Life $e_{x}^{o}$ : implies the average number of additional years a person aged $\mathrm{x}$ is expected to live under the prevailing mortality condition. It is defined as follows

$$
\mathrm{e}_{\mathrm{x}}^{0}=\frac{\mathrm{T}_{\mathrm{x}}}{1_{\mathrm{x}}}=\mathrm{e}_{\mathrm{x}}+0.5
$$

Where $e_{\mathrm{x}}$ is the curate expectation of life which implies the average number of complete years of life lived, but thr cohort $l_{0}$ after 
age $\mathrm{x}$ by each age of $\mathrm{l}_{\mathrm{x}}$ persons attaining that age. It is given as:

$$
e_{x}=\frac{L_{x+1}+L_{x+2}+\ldots+L_{w}}{1_{x}}
$$

It is related to the probability of survivorship by the following relation.

$$
P_{x}=\frac{e_{x}}{1+e_{x}}
$$

Where $P_{x}$ is the survival rate which is the probability between two complete years, and is define as:

$$
L_{X}=\frac{L_{X+1}}{L_{X}}
$$

In construction of life table, the death must be the only factor causing the number of cohort at various ages to decrease; the cohort originates from some standard number at birth say 10,000, 100,000 or 1,000,000 which is called the radix of life table [6]. Individuals die according to pre-determined mortality schedule at each age which is fixed and unchanged and deaths are uniformly distributed between one birthday and the next. These are some few specified assumptions used in constructing life table.

\section{Conclusion}

Life table is design essentially for the purpose of measuring survivorship, life expectation and mortality. It readily permits making mortality allowance for age cohort and eliminating the burden of compiling data on death for age cohort from the annual death statistics by age even when the letters are available. Life table is used by actuaries, demographers and many others to study the reproduction, migration, fertility and population growth. It is also used to make relative comparison of various measures of mortality such as death rate and expectations of life for two or more different groups of population. The table is accepted widely as an important tool in demographic and public health studies. That is why William farr referred life table to "The barometer of population", which is use to make projection of population size and characteristics.

\section{Acknowledgement}

None.

\section{Conflict of interest}

None.

\section{References}

1. Jose M P, Francisco M, Josep L. Introducing migratory flows in life table construction. 2012(36).

2. Bell, Felicitie C, Miller, Michael L. Life tables for the united states social security area $1900-2100 ; 2002$.

3. Bhagawati B, Choudhury L. Generation life table for India, 1901-1951. Middle East Journal of Age and Ageing. 2015;6(5):1-14.

4. Cox DR. Regression models and life -tables. Journal of the Royal Statistical Society. 2007;34(2):187-220.

5. Denton FT, Feaver CH, Spencer BG. Social and economic dimensions of an aging population. Sedap research. 2009; 247(432):1-26.

6. Arias E. National vital statistics reports united states life tables, 2010:63.2014.

7. Yang Z, Sun X. SAS R Macros for generating abridged and cause -eliminated life tables. 2008.

8. Scherbov S, Ediev D. Significance of life table estimates for small populations : Simulation-based study of standard errors. Demographic Research. 2011;24(22):527-550. 